\title{
Drug-resistant Enterobacteriaceae colonization is associated with healthcare utilization and antimicrobial use among inpatients in Pune, India
}

Renu Bharadwaj ${ }^{1,2^{*+}}$ D , Matthew L Robinson ${ }^{3+}$, Usha Balasubramanian ${ }^{1}$, Vandana Kulkarni ${ }^{1}$, Anju Kagal ${ }^{1,2}$, Priyanka Raichur ${ }^{1}$, Sandhya Khadse ${ }^{1,2}$, Dileep Kadam ${ }^{1,2}$, Chhaya Valvi ${ }^{1,2}$, Aarti Kinikar ${ }^{1,2}$, Savita Kanade ${ }^{1}$, Nishi Suryavanshi', Ivan Marbaniang ${ }^{1}$, George Nelson ${ }^{4}$, Julia Johnson ${ }^{3}$, Jonathan Zenilman ${ }^{3}$, Jonathan Sachs ${ }^{5}$, Amita Gupta ${ }^{1,3}$ and Vidya Mave $e^{1,3}$

\begin{abstract}
Background: Healthcare exposure may increase drug-resistant Enterobacteriaceae colonization risk. Nascent antimicrobial stewardship efforts in low- and middle-income countries require setting-specific data. We aimed to evaluate risk factors for inpatient drug resistant Enterobacteriaceae colonization in a resource-limited setting in India.
\end{abstract}

Methods: Patients age $\geq 6$ months admitted with $\geq 24 \mathrm{~h}$ of fever to a tertiary hospital in Pune, India were enrolled in a prospective cohort. Perirectal swabs, collected on admission and hospitalization day 3 or 4, were cultured in vancomycin- and ceftriaxone-impregnated media to assess for ceftriaxone-resistant Enterobacteriaceae (CTRE) and carbapenem-resistant Enterobacteriaceae (CPRE). Multivariable analyses assessed risk factors for drug-resistant Enterobacteriaceae colonization among participants without admission colonization.

Results: Admission perirectal swabs were collected on 897 participants; 87 (10\%) had CTRE and 14 (1.6\%) had CPRE colonization. Admission CTRE colonization was associated with recent healthcare contact $(p<0.01)$. Follow-up samples were collected from 620 participants, 67 (11\%) had CTRE and 21 (3.4\%) had CPRE colonization. Among 561 participants without enrollment CTRE colonization, 49 (9\%) participants were colonized with CTRE at follow-up. Detection of CTRE colonization among participants not colonized with CTRE at admission was independently associated with empiric third generation cephalosporin treatment (adjusted odds ratio [OR] 2.9, 95\% Cl 1.5-5.8). Follow-up transition to CPRE colonization detection was associated with ICU admission (OR 3.0, 95\% Cl 1.0-8.5).

Conclusions: Patients who receive empiric third generation cephalosporins and are admitted to the ICU rapidly develop detectable CTRE and CPRE colonization. Improved antimicrobial stewardship and infection control measures are urgently needed upon hospital admission.

Keywords: Antimicrobial resistance, Bacterial colonization, Antibiotic use, India, Prospective study

\footnotetext{
* Correspondence: renu.bharadwaj@gmail.com

${ }^{\dagger}$ Renu Bharadwaj and Matthew Robinson contributed equally to this work.

${ }^{1}$ Byramjee Jeejeebhoy Government Medical College-Johns Hopkins

University Clinical Research Site, Pune, Maharashtra, India

${ }^{2}$ Byramjee-Jeejeebhoy Government Medical College, Pune, Maharashtra, India

Full list of author information is available at the end of the article
}

(c) The Author(s). 2018 Open Access This article is distributed under the terms of the Creative Commons Attribution 4.0 International License (http://creativecommons.org/licenses/by/4.0/), which permits unrestricted use, distribution, and reproduction in any medium, provided you give appropriate credit to the original author(s) and the source, provide a link to the Creative Commons license, and indicate if changes were made. The Creative Commons Public Domain Dedication waiver (http://creativecommons.org/publicdomain/zero/1.0/) applies to the data made available in this article, unless otherwise stated. 


\section{Background}

Antibiotic use is increasing worldwide and has been implicated in the dramatic rise of antimicrobial resistance, which in turn threatens to reverse historical reductions in mortality for infectious diseases [1]. Few treatment options remain, for example, for Enterobacteriaceae, which are increasingly resistant to beta-lactam antibiotics due to production of extended spectrum beta-lactamase (ESBL), $\mathrm{AmpC}$, and carbapenemases. Infections with drug-resistant Enterobacteriaceae, such as those producing ESBL, have been associated with increased mortality [2].

The gut serves as a reservoir for drug-resistant Enterobacteriaceae [3]. Antibiotic administration increases drug-resistant Enterobacteriaceae colonization through selection pressure and disruption of protective normal microbiota [3, 4]. Animal models show a disruption to the gut microbiome within $12 \mathrm{~h}$ and emergence of drug resistance genes within 3 days of antibiotic administration $[5,6]$. Patients colonized with ESBL-producing Enterobacteriaceae are at greater risk for clinical infections with ESBL-producing Enterobacteriaceae than those who are not colonized [7].

In resource-limited settings, colonization with drug-resistant Enterobacteriaceae is common in adults, and, in community-based populations, associated with antibiotic use [8-10]. However, risk factors for inpatient acquisition of colonization with drug-resistant Enterobacteriaceae have not been clearly defined. As the world's largest consumer of antibiotics [11], India has among the highest burdens of antimicrobial resistance worldwide [12-14]. Travelers from high income countries to India return home colonized with ESBL-producing Enterobacteriaceae more frequently than travelers to other countries, suggesting that India has a heavier burden of drug-resistant Enterobacteriaceae colonization compared to other low and middle income countries $[15,16]$.

In this study, we sought to assess the clinical factors associated with ceftriaxone- and carbapenem-resistant Enterobacteriaceae colonization among adults and children admitted to a tertiary care hospital in Pune, India. Given prior work suggesting an association with antibiotic use, we enrolled patients with acute febrile illness, a group likely to be treated with antibiotics [17].

\section{Methods}

\section{Setting and participants}

Between August 2013 and December 2015, we prospectively enrolled adults and children admitted to medicine and pediatric wards with acute febrile illness at Byramjee Jeejeebhoy Government Medical College - Sassoon General Hospital, Pune, India, to assess antimicrobial resistance as previously reported [18]. Sassoon General Hospital is a 1300 bed public teaching hospital in Pune, a densely populated city in Maharashtra, India with a metropolitan population exceeding 5 million. We included patients greater than 6 months of age, with self-reported or measured fever $\geq 38.0{ }^{\circ} \mathrm{C}$ of more than $24 \mathrm{~h}$ duration who were screened within 1 day of admission. We excluded inpatient transfers from other hospitals, minor-age orphans, and medical-legal cases. A dedicated study physician and social worker obtained a standardized clinical and social history at the time of enrollment. After discharge, or on day 7 of enrollment, the study physician reviewed medication administration records and laboratory investigation results from the medical record.

\section{Sample collection and processing}

Between September 2014 and November 2015, a dedicated study nurse collected perirectal swabs on admission and on day 3 or 4 of hospitalization. Swabs were stored at $-80{ }^{\circ} \mathrm{C}$ pending processing $[19,20]$. Swabs were placed into peptone broth impregnated with ceftriaxone and vancomycin. After incubating for $24 \mathrm{~h}$, samples were plated onto MacConkey and sheep blood agar and incubated at $37^{\circ} \mathrm{C}$ for $18 \mathrm{~h}$. Isolates observed were loaded onto a Phoenix ${ }^{\ominus}$ Automated Microbiology System (Becton Dickinson) according to the manufacturer's instructions. The Phoenix ${ }^{\oplus}$ Automated Microbiology System performs species identification and drug susceptibility testing using a cartridge-based, broth microdilution system with a redox growth indicator [21].

\section{Definitions}

According to local hospital practice, patients $<12$ years of age are considered children and admitted to the pediatric ward, and those $\geq 12$ years of age, adolescents and adults are admitted to the medicine ward. Resistance to individual antibiotics was determined using Clinical Laboratory Standards Institute (CLSI) guidelines (2014-2015) reported by the Phoenix ${ }^{\odot}$ system [21]. Gram-negative isolates that grew in the presence of ceftriaxone impregnated broth were considered to be ceftriaxone-resistant, with the exception of Pseudomonas isolates. ESBL- producing organisms were identified using the Phoenix ${ }^{\oplus}$ system, which employs rules to adjudicate the presence or absence of ESBL based on susceptibility to five cephalosporins alone or in combination with the beta-lactamase inhibitor, clavulanic acid $[21,22]$. Enterobacteriaceae resistant to any carbapenem were considered to be carbapenem-resistant Enterobacteriaceae (CPRE) according to United States Centers for Disease Control and Prevention guidelines [23]. As defined elsewhere, drug-resistant Enterobacteriaceae colonization was defined as hospital-acquired if samples were negative at enrollment and positive at $>48 \mathrm{~h}$ of study [24, 25]. 


\section{Analysis}

Categorical variables were assessed for individual association with initial colonization, transition to colonization with drug-resistant Enterobacteriaceae isolates, and mortality using Fisher's exact test. The Wilcoxon rank sum test was used to assess length of hospital stay. Age was analyzed as a categorical variable stratified into five groups. A multivariable model was constructed assessing the association of predictor variables with acquisition of ceftriaxone-resistant Enterobacteriaceae (CTRE) colonization, adjusted for admission to the intensive care unit (ICU), sex, and age. Logistic regression was performed to assess predictor variables associated with all-cause mortality. Given the small sample size of CPRE colonization events, only bivariable analyses were performed. Statistical analyses were performed using $\mathrm{R}$ software [26].

\section{Results}

\section{Study population}

Between September 2014 and October 2015, 29,146 patients were admitted to adult and pediatric wards, 3589 had admission diagnoses suggestive of febrile illness, 1943 had subjective or documented fever, 1010 met all eligibility criteria and were enrolled in the parent study, 897 (89\%) participants were enrolled in the substudy and had baseline perirectal swabs collected. Of the 897 study participants, 358 (40\%) were children less than 12 years of age, 538 (60\%) were male, 131 (15\%) had been hospitalized within the past 3 months, and 214 (24\%) reported using antibiotics in the month prior to admission (Table 1).

Drug-resistant Enterobacteriaceae colonization at admission Of 897 participants, 91 (10\%) demonstrated growth of a Gram-negative rod (GNR) in ceftriaxone-impregnated media at admission, 87 (9.7\%) demonstrated growth of an Enterobacteriaceae. Colonization with CTRE at enrollment was higher in participants who had recent contact with the healthcare system including recently hospitalization, outpatient visit prior to hospitalization, or self-reported antibiotic use in the last month (Table 1). CPRE were found in 14 (1.6\%) participants on admission - 10 (1.9\%) adults and four $(1.1 \%)$ children. An additional four (0.4\%) participants grew Enterobacteriaceae with intermediate carbapenem susceptibility (Additional file 1: Table S1).

Drug-resistant Enterobacteriaceae colonization at follow-up A total of 620 (69\%) participants had follow-up swabs collected - 530 on Day 3 and 90 on Day 4. Of 277 (31\%) participants in whom a follow-up swab could not be

Table 1 Demographics and clinical characteristics of patients with and without ceftriaxone-resistant Enterobacteriaceae colonization at enrollment

\begin{tabular}{|c|c|c|c|}
\hline \multirow[t]{2}{*}{ Patient characteristic } & \multicolumn{2}{|c|}{ Colonization with ceftriaxone-resistant Enterobacteriaceae, $\mathrm{n}(\%)$ or median (IQR) } & \multirow{2}{*}{$\begin{array}{l}p- \\
\text { value }\end{array}$} \\
\hline & $\begin{array}{l}\text { Not colonized, } \\
n=810\end{array}$ & $\begin{array}{l}\text { Colonized, } \\
n=87\end{array}$ & \\
\hline Median age, years & $19(4-35)$ & $23(8-40)$ & 0.07 \\
\hline Children (age < 12 years) & $332(41)$ & $26(30)$ & 0.05 \\
\hline Male & $487(60)$ & $51(59)$ & 0.82 \\
\hline Diabetes & $34(4)$ & $5(6)$ & 0.42 \\
\hline HIV & $85(19)$ & $11(20)$ & 0.86 \\
\hline Alcoholism & $57(7)$ & $1(1)$ & 0.04 \\
\hline Smoking & $67(8)$ & $7(8)$ & 1.00 \\
\hline Income $<5000$ INR per month ${ }^{a}$ & $281(35)$ & $23(26)$ & 0.15 \\
\hline Works with animals ${ }^{a}$ & $150(19)$ & $20(23)$ & 0.31 \\
\hline Farmer or laborer $^{a}$ & $317(39)$ & $32(37)$ & 0.73 \\
\hline General practitioner visit prior to hospitalization & $273(36)$ & $37(44)$ & 0.19 \\
\hline Ayurvedic provider visit prior to hospitalization & $36(5)$ & $8(9)$ & 0.12 \\
\hline Hospitalized within the past 3 months & $113(14)$ & $18(21)$ & 0.11 \\
\hline Self-report of antibiotic use in the last month & $188(23)$ & $26(30)$ & 0.19 \\
\hline Recent healthcare contact ${ }^{\mathrm{b}}$ & $456(56)$ & $65(75)$ & $<0.01$ \\
\hline Admission to ICU & $153(19)$ & $13(15)$ & 0.47 \\
\hline Cough & $370(46)$ & $32(37)$ & 0.14 \\
\hline Diarrhea & $154(19)$ & $16(18)$ & 1.00 \\
\hline
\end{tabular}

IQR interquartile range, INR Indian rupees, ICU intensive care unit

a For children, this refers to the parents

${ }^{\mathrm{b} C o m p o s i t e ~ o f ~ v i s i t ~ t o ~ g e n e r a l ~ p r a c t i t i o n e r ~ o r ~ a y u r v e d i c ~ p r o v i d e r, ~ h o s p i t a l i z a t i o n ~ w i t h i n ~ t h e ~ p a s t ~} 3$ months, or self-reported antibiotic use in the last month 
collected, 212 had a hospital stay of 3 days or less (157 were discharged, 31 left against medical advice, 24 died), 30 refused collection of a second sample, and for 35 a second sample could not be obtained for other reasons (Fig. 1). Participants without a follow-up swab were more likely to be adults ( $78 \%$ vs $52 \%, p<0.01$ ) but were less likely to have HIV ( $12 \%$ vs $23 \%, p<0.01)$, to have been admitted to the hospital within the past 3 months ( $11 \%$ vs $16 \%, p<0.04)$, or to have been admitted to the intensive care unit (ICU) $(13 \%$ vs $21 \%, p<0.01)$. There was no difference, however, in the proportion of participants receiving antibiotics ( $82 \%$ vs $85 \%, p=0.23)$, including third generation cephalosporins $(49 \%$ vs $48 \%, p=$ 0.89). Of 620 participants, 78 (13\%) demonstrated growth of a GNR; 67 (11\%) were identified as growing CTRE. CPRE were found in 21 (3.4\%) participants including 8 (2.5\%) adults and 13 (4.4\%) children; 2 (0.3\%) participants grew Enterobacteriaceae isolates with intermediate carbapenem susceptibility.

Of 561 (90\%) participants without baseline resistant Enterobacteriaceae colonization, $49(9 \%)$ were found to have colonization at follow-up (Table 2). Extremes of age were associated with increased likelihood of CTRE colonization acquisition. Empiric therapy with a third generation cephalosporin was also associated with acquisition of CTRE colonization (71\% received cephalosporin vs. $46 \%$ did not, $\mathrm{p}<0.01$ ) (Table 2). In multivariable analysis, adjusting for age, sex, ICU admission, and third generation cephalosporin initial antibiotic prescription, advanced age and third generation cephalosporin use remained significantly associated with acquisition of CTRE colonization, with cephalosporin use demonstrating nearly 3 times higher odds of acquisition (adjusted OR 2.9, 95\% CI 1.5-5.8) (Table 2).

There were 19 (3\%) participants (13 adults and 6 children) who on admission were not colonized with CPRE, but were found to have CPRE colonization on follow-up. Children under age 5 were most likely to acquire CPRE colonization compared to other age categories (OR 4.1, 95\% CI 1.3-18.4). Participants who acquired CPRE were more likely to be admitted to the ICU (OR 3.0, 95\% CI 1.0-8.5) and more likely to have received empiric aminoglycosides (OR 4.2, 95\% CI 1.2-4.3). There was notably an association between ICU admission and aminoglycoside use, $p<0.001$.

Among 59 (9\%) participants found to have CTRE colonization at enrollment in whom follow-up samples were collected, colonization remained detectable in 22 (37\%) participants (Fig. 1). CTRE Use of third-generation cephalosporins was not associated with loss of detection of CTRE colonization $(p=0.79)$. Among the 14 participants

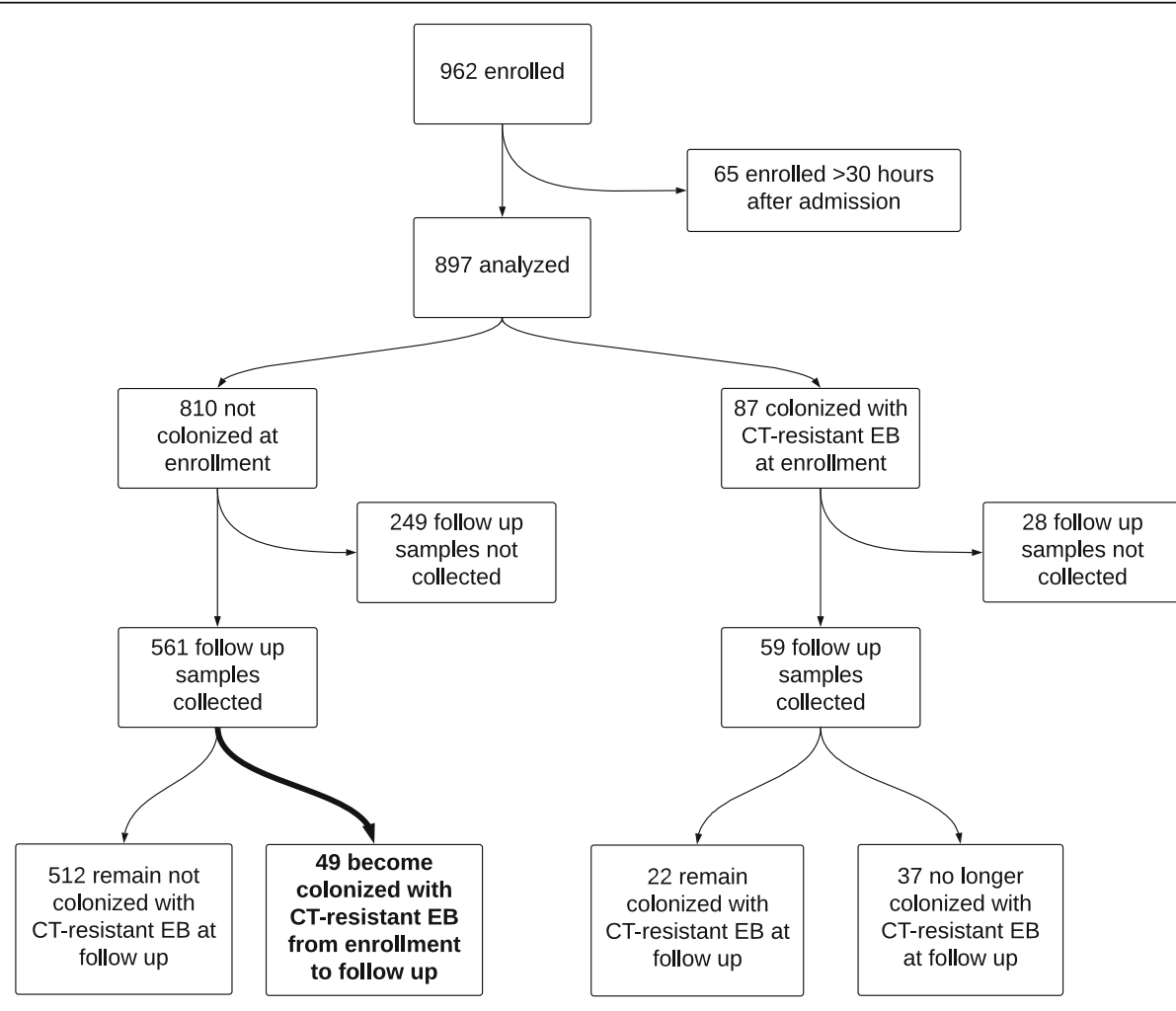

CT: ceftriaxone; EB: Enterobacteriaceae

Fig. 1 Acquisition of ceftriaxone-resistant Enterobacteriaceae colonization. Abbreviations - CTX: ceftriaxone; EB: Enterobacteriaceae 
Table 2 Factors associated with detection of ceftriaxone-resistant Enterobacteriaceae colonization among 561 patients without ceftriaxone-resistant Enterobacteriaceae colonization at enrollment

\begin{tabular}{|c|c|c|c|c|c|c|}
\hline \multirow[t]{2}{*}{ Risk Factor, n (\%) } & \multicolumn{2}{|c|}{ Ceftriaxone-resistant Enterobacteriaceae colonization, n (\%) } & \multirow{2}{*}{$\begin{array}{l}\text { Unadjusted } \\
\text { OR (95\% Cl) }\end{array}$} & \multirow{2}{*}{$\begin{array}{l}p- \\
\text { value }\end{array}$} & \multirow{2}{*}{$\begin{array}{l}\text { Adjusted } \\
\mathrm{OR}^{\mathrm{a}}(95 \% \mathrm{Cl})\end{array}$} & \multirow{2}{*}{$\begin{array}{l}p- \\
\text { value }\end{array}$} \\
\hline & $\begin{array}{l}\text { No acquisition, } \\
N=512\end{array}$ & $\begin{array}{l}\text { Acquisition, } \\
N=49\end{array}$ & & & & \\
\hline \multicolumn{7}{|l|}{ Age in years } \\
\hline$<5$ & $163(32)$ & $21(43)$ & $4.7(1.3-29.9)$ & 0.04 & $4.4(1.2-28.7)$ & 0.06 \\
\hline $5-11$ & $88(17)$ & $5(10)$ & $2.1(0.4-14.8)$ & 0.39 & $2.0(0.4-14.6)$ & 0.41 \\
\hline $12-23$ & $73(14)$ & $2(4)$ & Ref & Ref & Ref & Ref \\
\hline $24-59$ & $154(30)$ & $15(31)$ & $3.6(1-22.9)$ & 0.1 & $3.8(1-24.9)$ & 0.08 \\
\hline$\geq 60$ & $34(7)$ & $6(12)$ & $6.4(1.4-45.5)$ & 0.03 & $7.0(1.5-50.1)$ & 0.02 \\
\hline Male sex & $307(60)$ & $33(67)$ & $1.4(0.7-2.8)$ & 0.36 & $1.5(0.8-2.9)$ & 0.23 \\
\hline Income $<5000$ INR / month & $183(36)$ & $23(47)$ & $1.6(0.0-8-3)$ & 0.12 & & \\
\hline Farmer or laborer & $203(40)$ & $24(49)$ & $1.5(0.8-2.7)$ & 0.22 & & \\
\hline$H I V^{b}$ & $56(22)$ & $6(27)$ & $1.3(0.4-3.7)$ & 0.6 & & \\
\hline $\mathrm{ICU}$ & $102(20)$ & $17(35)$ & $2.1(1.1-4.1)$ & 0.03 & $1.6(0.8-3.4)$ & 0.18 \\
\hline \multicolumn{7}{|l|}{ Pre-hospital antibiotic use } \\
\hline Within the past week & $99(19)$ & $13(27)$ & $1.5(0.7-3.0)$ & 0.26 & & \\
\hline Within the past month & $121(24)$ & $14(29)$ & $1.3(0.6-2.6)$ & 0.48 & & \\
\hline \multicolumn{7}{|l|}{ Initial inpatient antibiotic Rx } \\
\hline Any antibiotic & $432(84)$ & $45(92)$ & $2.1(0.7-8.2)$ & 0.21 & & \\
\hline Multiple antibiotics & $240(47)$ & $30(61)$ & $1.8(0.9-3.5)$ & 0.07 & & \\
\hline 3rd-gen cephalosporin & $234(46)$ & $35(71)$ & $3.0(1.5-6.1)$ & $<.01$ & $2.9(1.5-5.8)$ & $<0.01$ \\
\hline Fluoroquinolone & $19(4)$ & $1(2)$ & $0.5(0.0-3.6)$ & 1 & & \\
\hline Macrolide & $96(19)$ & $13(27)$ & $1.6(0.7-3.2)$ & 0.19 & & \\
\hline Aminoglycoside & $53(10)$ & $8(16)$ & $1.7(0.6-3.9)$ & 0.23 & & \\
\hline
\end{tabular}

$O R$ odds ratio, $I Q R$ interquartile range, $I C U$ intensive care unit, INR Indian rupees, $R x$ prescription

${ }^{a}$ Adjusted for age, sex, ICU admission, and third generation cephalosporin initial antibiotic prescription

${ }^{b}$ HIV test results were available for 249 patients without ceftriaxone-resistant Enterobacteriaceae acquisition and 22 patients with ceftriaxone-resistant

Enterobacteriaceae acquisition

found to have CPRE colonization at enrollment, follow-up samples were collected on 12 participants, of which two (17\%) continued to demonstrate CPRE colonization.

\section{Perirectal isolate species and antimicrobial resistance patterns}

The 136 participants with CTRE identified in either enrollment or follow-up samples comprised $52(14 \%)$ of the enrolled children and 84 (16\%) of the enrolled adults. Five specimens grew two different CTRE species. Of the 101 ceftriaxone-resistant Escherichia coli isolates, 77 (76\%) were identified as ESBL producers by Phoenix ${ }^{\circ}$, while 32 of 49 (65\%) Klebsiella isolates were identified as ESBL producers (Table 3). Though the Phoenix ${ }^{\circ}$ system does not report the presence of AmpC as part of the resistance profile for identified organisms, cefoxitin resistance may suggest the presence of $\mathrm{AmpC}$, particularly in the context of a ceftriaxone-resistant organism that is not flagged as an ESBL organism by Phoenix ${ }^{\circ}$. Both E. coli and Klebsiella species were mostly resistant to cefoxitin -66 and $63 \%$ respectively. None of the 11 Enterobacter isolates were considered to be ESBL isolates by Phoenix ${ }^{\circ}$, and all were resistant to cefoxitin, suggesting that $\mathrm{AmpC}$ was the likely mechanism of resistance for the Enterobacter isolates.

\section{Clinical outcomes}

The median length of stay among the 897 participants was 4.0 days, interquartile range (IQR) (3-7); 4.0 days (IQR 2-6) for adults and 5.5 days (IQR 4-9) for children. Among participants who had follow-up samples collected, median length of stay was 6.0 days (IQR 4-9). There was no association with length of stay and Enterobacteriaceae colonization at follow-up, $p=0.35$. There were 34 deaths (6\%) among participants with collected follow-up samples. Participants who were colonized at follow-up were more likely to die. Among 71 participants who were colonized with CTRE at follow-up there were 8 (11\%) deaths, whereas among 549 participants who were not colonized there were $26(5 \%)$ deaths $(p=0.02)$ (Table 4$)$. In a multivariable model adjusted for age $<12$ years and admission 
Table 3 Isolate species and resistance pattern among 179 Gram-negative perirectal isolates

\begin{tabular}{|c|c|c|c|c|}
\hline Species & n (\%) & ESBL, n (\%) & Cefoxitin resistant, n (\%) & Carbapenem resistant, $\mathrm{n}(\%)$ \\
\hline All isolates & $179(100)$ & - & - & $38(21)$ \\
\hline Pseudomonas species & $14(8)$ & - & - & $2(14)$ \\
\hline Comamonas species & $2(1)$ & - & - & 0 \\
\hline Moraxella species & $1(0.6)$ & - & - & 0 \\
\hline All Enterobacteriaceae & $162(91)$ & $109(67)$ & $110(68)$ & $36(22)$ \\
\hline Escherichia coli & $101(78)$ & $77(76)$ & $67(66)$ & $16(15)$ \\
\hline Klebsiella pneumoniae & $49(27)$ & $32(65)$ & $31(63)$ & $16(33)$ \\
\hline Enterobacter species & $11(6)$ & 0 & $11(100)$ & $3(27)$ \\
\hline Citrobacter farmeri & $1(0.6)$ & 0 & $1(100)$ & $1(100)$ \\
\hline
\end{tabular}

ESBL extended spectrum beta-lactamase

to the ICU, CTRE colonization was no longer associated with mortality (AOR 2.4, 95\% CI 0.9-6.0) (Table 4). There were three (9\%) deaths among participants who were colonized with CPRE at enrollment or follow-up.

\section{Discussion}

Our study has several key findings. First, we identified a 10\% prevalence of community-acquired ceftriaxone-resistant GNR colonization and 1.6\% colonization with CPRE among Indian adults and children admitted with acute febrile illness. Second, we observed that healthcare contact was associated with increased odds of admission CTRE colonization. Third, we found that participants without baseline CTRE colonization who received empiric third generation cephalosporins had almost three-fold higher odds of follow up detection of CTRE colonization, and were more likely to acquire CPRE colonization if admitted to the ICU.

The $10 \%$ rate of community-acquired CTRE colonization reported in this study is comparable to rates of $10-15 \%$ reported in studies that used rectal and perirectal swabs to detect colonization [27, 28], and to another study of children in India showing 13\% of children to have ceftriaxone-resistant E. coli colonization [29]. The rate was lower than the 23-69\% ESBL-Enterobacteriaceae colonization rate reported among healthy adults from other resource limited Asian settings that determined colonization using higher inoculum stool samples $[8,9]$. While obtaining a stool specimen from healthy volunteers at a time of convenience may be practical for community surveillance studies, in order to assess dynamic colonization during hospitalization, perirectal swab collection at specific time intervals is more practical than stool culture.

Admission CTRE colonization was no longer detected at follow-up for almost two-thirds of CTRE colonized participants, a higher than expected proportion. Although some studies have shown persistence of multidrug-resistant Enterobacteriaceae for months after acquisition, others have shown that with daily screening, more than half of patients demonstrate intermittent colonization [25, 30, 31]. As we only performed one follow-up culture, it is possible that

Table 4 Factors associated with mortality among patients who completed follow-up $(N=580)^{\text {a }}$

\begin{tabular}{|c|c|c|c|c|c|c|}
\hline Clinical Factor & $\begin{array}{l}\text { Survived, } N=546, \\
\mathrm{n}(\%)\end{array}$ & $\begin{array}{l}\text { Died, } N=34, \\
\mathrm{n}(\%)\end{array}$ & $\begin{array}{l}\text { Unadjusted OR } \\
(95 \% \mathrm{Cl})\end{array}$ & $p$-value & $\begin{array}{l}\text { Adjusted OR } \\
(95 \% \mathrm{Cl})\end{array}$ & $\overline{p \text {-value }}$ \\
\hline Male sex & $321(59)$ & $23(68)$ & $1.5(0.7-3.4)$ & 0.37 & - & - \\
\hline Child $<12$ years & $267(49)$ & $11(32)$ & $0.5(0.2-1.1)$ & 0.08 & $0.2(0.1-0.4)$ & $<0.01$ \\
\hline Income < 5000 INR / month & $197(36)$ & $15(44)$ & $1.4(0.6-3)$ & 0.36 & - & - \\
\hline Diabetes & $18(3)$ & $1(3)$ & $0.9(0-6)$ & 1 & - & - \\
\hline HIV & $56(10)$ & $7(21)$ & $2(0.6-5.7)$ & 0.17 & - & - \\
\hline Diarrhea & $110(20)$ & $9(26)$ & $1.4(0.6-3.3)$ & 0.38 & - & - \\
\hline Cough & $278(51)$ & $13(38)$ & $0.6(0.3-1.3)$ & 0.16 & - & - \\
\hline ICU admission & $96(18)$ & $19(56)$ & $6.8(3.1-15.6)$ & $<.01$ & $14.7(6.1-36.9)$ & $<0.01$ \\
\hline Admission ceftriaxone-resistant EB colonization & $52(10)$ & $2(6)$ & $0.6(0.1-2.4)$ & 0.76 & - & - \\
\hline Follow-up ceftriaxone-resistant EB colonization & $53(10)$ & $8(24)$ & $2.9(1.1-6.9)$ & 0.02 & $2.4(0.9-6.0)$ & 0.06 \\
\hline Acquisition of ceftriaxone-resistant EB colonization & $37(7)$ & $6(18)$ & $2.8(0.9-7.7)$ & 0.04 & - & - \\
\hline
\end{tabular}

$O R$ odds ratio, INR Indian Rupees, ICU intensive care unit, EB Enterobacteriaceae

${ }^{a}$ Mortality data was not available for $40(6 \%)$ of patients who completed follow-up perirectal swab collection

${ }^{\mathrm{b}}$ Adjusted for child $<12$ years, ICU admission, and colonization with ceftriaxone-resistant EB at follow-up 
further follow-up cultures may have revealed persistence of colonization in additional participants. However, because third-generation cephalosporin use was not associated with loss of detectable ceftriaxone-resistant GNR colonization, limitations in the CTRE colonization detection modality were not impacted by third-generation cephalosporin use.

Use of empiric third generation cephalosporins was associated with detection of CTRE colonization among participants not found to be colonized at admission, even when adjusted for other factors. Empiric aminoglycosides use was associated with acquisition of carbapenem-resistant Enterobacteriaceae colonization, but was likely confounded by ICU admission. From this study, we cannot determine if follow-up detection of drug-resistant Enterobacteriaceae colonization among participants without baseline colonization was due to nosocomial transmission or overgrowth of minority drug-resistant colonies in the presence of selection pressure from administered antibiotics. Most nosocomial acquisition of drug-resistant Enterobacteriaceae in studies conducted in resource-rich settings is not attributed to transmission from other hospitalized patients $[32,33]$. However, a study in a resource-limited setting showed genetic similarities among acquired isolates suggesting nosocomial cross-transmission [34].

Previous work in a resource-rich ICU setting has shown an association between drug-resistant Enterobacteriaceae colonization and mortality [35], but similar data from resource-limited settings describing clinical associations with colonization is limited. We found that participants with CTRE colonization at follow-up were more likely to die in an unadjusted analysis. After adjusting for ICU admission and age, the association no longer remained statistically significant. Any association of CTRE colonization and mortality does not necessarily signify causality, as there may be unmeasured risk factors common to both CTRE colonization acquisition and mortality.

Unfettered antibiotic use has been implicated as one of the key factors driving global increasing antimicrobial resistance [1]. Studies in other settings have demonstrated that antibiotic administration and ICU admission are associated with development of antibiotic-resistant bacterial stool colonization during the course of a hospitalization, but used longer intervals between enrollment and follow-up $[28,36]$. The finding that initial empiric antibiotic choice can also significantly impact this process is concerning, especially given how commonly third generation cephalosporins are used in India and elsewhere.

Antimicrobial use may be tempered by antimicrobial stewardship policies, which were not yet in place at the time of this study. Guidelines offer two general antimicrobial stewardship approaches: preauthorization of antibiotics and auditing of antibiotic prescriptions after treatment initiation [37, 38]. De-escalation of antibiotic therapy was associated in a meta-analysis with decreased mortality risk [39]. However, it remains unclear how quickly antibiotics should be de-escalated. Rapid acquisition of detectable resistant Enterobacteriaceae colonization, as noted in our study, may result in a narrow window of opportunity for prevention of drug-resistant organism colonization. A recent study notably failed to show a reduction in the emergence of multidrug-resistant Gram-negative infections after de-escalation of anti-pseudomonal beta-lactams [40]. Our study design required participants to remain hospitalized through Day 3 or Day 4, which excluded both those with early mortality, and those with mild illness who were quickly discharged. However, among patients who do remain hospitalized through the third or fourth day of admission, the contribution of empiric antibiotics to drug-resistant Enterobacteriaceae colonization poses an antimicrobial stewardship concern.

\section{Conclusions}

In conclusion, colonization with drug-resistant Enterobacteriaceae is common among patients admitted with fever in Pune, India. Our study highlights the need for improved antimicrobial stewardship and infection control measures, which the World Health Organization acknowledges in its Global Action Plan on Antimicrobial Resistance [41]. Physicians and governing bodies in India have recognized the threat of antimicrobial resistance responding with newly introduced legislation which requires a prescription for the sale of many advanced antibiotics including third and fourth generation cephalosporins [12, 42]. Continued surveillance of drug-resistant Enterobacteriaceae colonization in India and other resource-limited settings is warranted.

\section{Additional file}

Additional file 1: Table S1. Demographics and clinical characteristics of patients with and without carbapenem-resistant Enterobacteriaceae colonization at enrollment. (PDF $43 \mathrm{~kb}$ )

\section{Abbreviations \\ CPRE: Carbapenem-resistant Enterobacteriaceae; CTRE: Ceftriaxone-resistant Enterobacteriaceae; EB: Enterobacteriaceae; ESBL: Extended spectrum beta- lactamase; GNR: Gram-negative rod; ICU: Intensive care unit; INR: Indian rupees; IQR: Interquartile range; OR: Odds ratio; Rx: Prescription}

\section{Acknowledgments}

We thank the study participants and staff for their immense contribution.

\section{Funding}

This study was supported by the Ujala Foundation, Newton Square, PA; Johns Hopkins Center for Innovative Medicine; Sacharuna Foundation; and Gilead Foundation. VM, NS, SK, UB, VK, IM and AG were also supported by the U.S. National Institutes of the Health (NIH) BWI HIV Clinical Trials Unit (U01 Al069497). MR and JS were supported by NIH Research Training Grant R25 TW009340 funded by the Fogarty International Center and the NIH Office of the Director Office of AIDS Research. MR was also supported by $\mathrm{NIH}$ 
Training Grant T32 Al007291. JJ was supported by NIH Training Grant T32 HL 125239-1. The content is solely the responsibility of the authors and does not necessarily represent the official views of the NIH. The funding sources had no role in study design, data collection, analysis, or writing.

\section{Availability of data and materials}

The datasets used and/or analyzed during the current study are available from the corresponding author on reasonable request.

\section{Authors' contributions}

$\mathrm{RB}$ contributed to study design, interpretation of data, and manuscript preparation. MR analyzed data and prepared the manuscript. UB and VK designed and performed the bacteriology procedures. AK1, NS, and GN contributed to study design and management. PR collected clinical data and interpreted interim data. SK2 assisted in study design and data collection. DK, CV, AK2, and SK1 oversaw clinical data collection and contributed to data interpretation. IM contributed to study management. JJ contributed to data interpretation and manuscript preparation. JZ contributed to study design. JS contributed to data collection and management. AG and VM oversaw study design, execution, analysis, and manuscript preparation. All authors read and approved the final manuscript.

\section{Ethics approval and consent to participate}

This study was approved by the BJGMC-SGH Ethics Committee and Institutional Review Board of Johns Hopkins University School of Medicine (Number NA_00078938). Written informed consent was obtained from adults 18 years and older, or by the legal guardian in the case of children under age 18 years. An additional assent was obtained from children 12 to 18 years of age.

\section{Consent for publication}

\section{Not applicable}

\section{Competing interests}

The authors declare that they have no competing interests.

\section{Publisher's Note}

Springer Nature remains neutral with regard to jurisdictional claims in published maps and institutional affiliations.

\section{Author details}

'Byramjee Jeejeebhoy Government Medical College-Johns Hopkins University Clinical Research Site, Pune, Maharashtra, India. ${ }^{2}$ Byramjee-Jeejeebhoy Government Medical College, Pune, Maharashtra, India. ${ }^{3}$ Johns Hopkins University School of Medicine, Baltimore, MD, USA. ${ }^{4}$ Vanderbilt University School of Medicine, Nashville, TN, USA. ${ }^{5}$ Phoenix Children's Hospital / Maricopa Medical Center, Phoenix, AZ, USA.

Received: 24 January 2018 Accepted: 17 September 2018 Published online: 04 October 2018

\section{References}

1. Holmes AH, Moore LS, Sundsfjord A, Steinbakk M, Regmi S, Karkey A, Guerin PJ, Piddock $L$. Understanding the mechanisms and drivers of antimicrobial resistance. Lancet. 2016;387(10014):176-87.

2. Schwaber MJ, Carmeli Y. Mortality and delay in effective therapy associated with extended-spectrum beta-lactamase production in Enterobacteriaceae bacteraemia: a systematic review and meta-analysis. J Antimicrob Chemother. 2007;60(5):913-20.

3. Donskey CJ. Antibiotic regimens and intestinal colonization with antibioticresistant gram-negative bacilli. Clin Infect Dis. 2006;43(Supplement 2):S62-9.

4. Stecher B, Hardt WD. Mechanisms controlling pathogen colonization of the gut. Curr Opin Microbiol. 2011;14(1):82-91.

5. Antunes LCM, Han J, Ferreira RBR, Lolić P, Borchers CH, Finlay BB. Effect of antibiotic treatment on the intestinal Metabolome. Antimicrob Agents Chemother. 2011;55(4):1494-503.

6. Looft T, Johnson TA, Allen HK, Bayles DO, Alt DP, Stedtfeld RD, Sul WJ, Stedtfeld TM, Chai B, Cole JR, et al. In-feed antibiotic effects on the swine intestinal microbiome. Proc Natl Acad Sci U S A. 2012;109(5):1691-6.

7. Vehreschild MJ, Hamprecht A, Peterson L, Schubert S, Hantschel M, Peter S, Schafhausen P, Rohde H, Lilienfeld-Toal MV, Bekeredjian-Ding I, et al. A multicentre cohort study on colonization and infection with ESBL- producing Enterobacteriaceae in high-risk patients with haematological malignancies. J Antimicrob Chemother. 2014;69(12):3387-92.

8. Stoesser N, Xayaheuang S, Vongsouvath M, Phommasone K, Elliott I, Del Ojo Elias C, Crook DW, Newton PN, Buisson Y, Lee SJ et al: Colonization with Enterobacteriaceae producing ESBLs in children attending pre-school childcare facilities in the Lao People's Democratic Republic. J Antimicrob Chemother 2015, 70(6):1893-1897.

9. Luvsansharav UO, Hirai I, Nakata A, Imura K, Yamauchi K, Niki M, Komalamisra C, Kusolsuk T, Yamamoto Y. Prevalence of and risk factors associated with faecal carriage of CTX-M beta-lactamase-producing Enterobacteriaceae in rural Thai communities. J Antimicrob Chemother. 2012;67(7):1769-74.

10. Pathak A, Chandran SP, Mahadik K, Macaden R, Stalsby Lundborg C. Frequency and factors associated with carriage of multi-drug resistant commensal Escherichia coli among women attending antenatal clinics in Central India. BMC Infect Dis. 2013;13:199.

11. Van Boeckel TP, Gandra S, Ashok A, Caudron Q, Grenfell BT, Levin SA, Laxminarayan R. Global antibiotic consumption 2000 to 2010: an analysis of national pharmaceutical sales data. Lancet Infect Dis. 2014;14(8):742-50.

12. Laxminarayan $R$, Chaudhury RR. Antibiotic resistance in India: drivers and opportunities for action. PLoS Med. 2016;13(3):e1001974.

13. Hawser SP, Bouchillon SK, Hoban DJ, Badal RE, Hsueh PR, Paterson DL. Emergence of high levels of extended-spectrum-beta-lactamase-producing gram-negative bacilli in the Asia-Pacific region: data from the study for monitoring antimicrobial resistance trends (SMART) program, 2007. Antimicrob Agents Chemother. 2009;53(8):3280-4.

14. World Health Organization: Antimicrobial resistance: global report on surveillance. 2014

15. Kuenzli E, Jaeger VK, Frei R, Neumayr A, DeCrom S, Haller S, Blum J, Widmer $A F$, Furrer $H$, Battegay $M$, et al. High colonization rates of extendedspectrum beta-lactamase (ESBL)-producing Escherichia coli in Swiss travellers to South Asia- a prospective observational multicentre cohort study looking at epidemiology, microbiology and risk factors. BMC Infect Dis. 2014;14(1):528.

16. Tängden $T$, Cars $O$, Melhus $A$, Löwdin E. Foreign travel is a major risk factor for colonization with Eschericha coli producing CTX-M-type extendedspectrum beta-lactamase: a prospective study with Swedish volunteers. Antimicrob Agents Chemother. 2010;54(9):3564-8.

17. Chandy SJ, S Kl, Jeyaseelan L, Kumar R, Suresh S: Antimicrobial prescription patterns for common acute infections in some rural \& urban health facilities of India. Indian J Med Res 2008, 128(2):165-171.

18. Mave V, Chandanwale A, Kagal A, Khadse S, Kadam D, Bharadwaj R, Dohe V, Robinson ML, Kinikar A, Joshi S, et al. High burden of antimicrobial resistance and mortality among adults and children with community-onset bacterial infections in India. J Infect Dis. 2017:215(8):1312-20.

19. Instructions for Collecting Stool Specimens https://www.cdc.gov/foodsafety/ outbreaks/investigating-outbreaks/specimen-collection.html. Accessed 1 May 2018

20. Dan M, Richardson J, Miliotis MD, Koornhof HJ. Comparison of preservation media and freezing conditions for storage of specimens of faeces. J Med Microbiol. 1989:28(2):151-4.

21. Carroll KC, Glanz BD, Borek AP, Burger C, Bhally HS, Henciak S, Flayhart D. Evaluation of the BD Phoenix automated microbiology system for identification and antimicrobial susceptibility testing of Enterobacteriaceae. J Clin Microbiol. 2006:44(10):3506-9.

22. Sanguinetti M, Posteraro B, Spanu T, Ciccaglione D, Romano L, Fiori B, Nicoletti G, Zanetti S, Fadda G. Characterization of clinical isolates of Enterobacteriaceae from Italy by the BD Phoenix extended-spectrum betalactamase detection method. J Clin Microbiol. 2003;41(4):1463-8.

23. Nora C, Sandra NB, Thiphasone K-T, Ruth L, Kristin MS, Paula Snippes V, Marion AK, Daniel BM, Lucy W, Elisabeth V, et al. Improved phenotypebased definition for identifying Carbapenemase producers among Carbapenem-resistant Enterobacteriaceae. Emerg Infect Dis. 2015;21(9):1611.

24. Barbier F, Pommier C, Essaied W, Garrouste-Orgeas M, Schwebel C, Ruckly S, Dumenil AS, Lemiale V, Mourvillier B, Clec'h C, et al. Colonization and infection with extended-spectrum beta-lactamase-producing Enterobacteriaceae in ICU patients: what impact on outcomes and carbapenem exposure? J Antimicrob Chemother. 2016:71(4):1088-97.

25. Grohs P, Podglajen I, Guerot E, Bellenfant F, Caumont-Prim A, Kac G, Tillecovidin B, Carbonnelle E, Chatellier G, Meyer G, et al. Assessment of five screening strategies for optimal detection of carriers of third-generation 
cephalosporin-resistant Enterobacteriaceae in intensive care units using daily sampling. Clin Microbiol Infect. 2014;20(11):0879-86.

26. R Core Team. R: a language and environment for statistical computing. Vienna: R Foundation for Statistical Computing; 2015.

27. Thiebaut AC, Arlet G, Andremont A, Papy E, Sollet JP, Bernede-Bauduin C, Guillemot D, Schlemmer B, ColoRea Study G. Variability of intestinal colonization with third-generation cephalosporin-resistant Enterobacteriaceae and antibiotic use in intensive care units. J Antimicrob Chemother. 2012;67(6):1525-36.

28. Razazi $K$, Derde LPG, Verachten M, Legrand P, Lesprit P, Brun-Buisson C. Clinical impact and risk factors for colonization with extended-spectrum beta-lactamase-producing bacteria in the intensive care unit. Intensive Care Med. 2012:38(11):1769-78.

29. Shakya P, Barrett P, Diwan V, Marothi Y, Shah H, Chhari N, Tamhankar AJ, Pathak A, Lundborg CS. Antibiotic resistance among Escherichia coli isolates from stool samples of children aged 3 to 14 years from Ujjain, India. BMC Infect Dis. 2013;13:477.

30. Apisarnthanarak A, Bailey TC, Fraser VJ. Duration of stool colonization in patients infected with extended-spectrum beta-lactamase-producing Escherichia coli and Klebsiella pneumoniae. Clin Infect Dis. 2008;46(8):1322-3.

31. Weintrob AC, Roediger MP, Barber M, Summers A, Fieberg AM, Dunn J, Seldon V, Leach F, Huang XZ, Nikolich MP, et al. Natural history of colonization with gram-negative multidrug-resistant organisms among hospitalized patients. Infect Control Hosp Epidemiol. 2010;31(4):330-7.

32. Kim J, Lee JY, Kim SI, Song W, Kim J-S, Jung S, Yu JK, Park KG, Park Y-J. Rates of fecal transmission of extended-Spectrum $\beta$-lactamase-producing and Carbapenem-resistant Enterobacteriaceae among patients in intensive care units in Korea. Ann Lab Med. 2014;34(1):20-5.

33. Tschudin-Sutter S, Frei R, Dangel M, Stranden A, Widmer AF. Rate of transmission of extended-Spectrum Beta-lactamase-producing Enterobacteriaceae without contact isolation. Clin Infect Dis. 2012;55(11): 1505-11.

34. Woerther PL, Angebault C, Jacquier H, Hugede HC, Janssens AC, Sayadi S, El Mniai A, Armand-Lefevre L, Ruppe E, Barbier F, et al. Massive increase, spread, and exchange of extended Spectrum beta-lactamase-encoding genes among intestinal Enterobacteriaceae in hospitalized children with severe acute malnutrition in Niger. Clin Infect Dis. 2011;53(7):677-85.

35. Dautzenberg MJ, Wekesa AN, Gniadkowski M, Antoniadou A, Giamarellou H, Petrikkos GL, Skiada A, Brun-Buisson C, Bonten MJ, Derde LP, et al. The association between colonization with carbapenemase-producing enterobacteriaceae and overall ICU mortality: an observational cohort study. Crit Care Med. 2015;43(6):1170-7.

36. Han JH, Bilker WB, Nachamkin I, Tolomeo P, Mao X, Fishman NO, Lautenbach $\mathrm{E}$. Impact of antibiotic use during hospitalization on the development of gastrointestinal colonization with Escherichia coli with reduced fluoroquinolone susceptibility. Infect Control Hosp Epidemiol. 2013; 34(10):1070-6

37. Timothy HD, Robert CO, John E, McGowan DNG Jr. Weinstein RA, Burke JP, Huskins WC, David LP, Neil OF, Christopher FC, et al. Infectious Diseases Society of America and the Society for Healthcare Epidemiology of America Guidelines for developing an institutional program to enhance antimicrobial stewardship. Clin Infect Dis. 2007;44(2):159-77.

38. Ashiru-Oredope D, Sharland M, Charani E, McNulty C, Cooke J, Group AAS Improving the quality of antibiotic prescribing in the NHS by developing a new antimicrobial stewardship Programme: start Smart--then focus. J Antimicrob Chemother. 2012;67(Suppl 1):i51-63.

39. Schuts EC, Hulscher ME, Mouton JW, Verduin CM, Stuart JW, Overdiek HW, van der Linden PD, Natsch S, Hertogh CM, Wolfs TF, et al. Current evidence on hospital antimicrobial stewardship objectives: a systematic review and meta-analysis. Lancet Infect Dis. 2016;16(7):847-56.

40. Bus L, Denys W, Catteeuw J, Gadeyne B, Vermeulen K, Boelens J, Claeys G, Waele JJ, Decruyenaere J, Depuydt PO. Impact of de-escalation of betalactam antibiotics on the emergence of antibiotic resistance in ICU patients: a retrospective observational study. Intensive Care Med. 2016:1-11.

41. World Health Organization: Global Action Plan on Antmicrobial Resistance. 2015.

42. Global Antibiotic Resistance Partnership (GARP) - India Working Group. Rationalizing antibioitc use to limit antibiotic resistance in India. Indian J Med Res. 2011;134:281-94.

\section{Ready to submit your research? Choose BMC and benefit from:}

- fast, convenient online submission

- thorough peer review by experienced researchers in your field

- rapid publication on acceptance

- support for research data, including large and complex data types

- gold Open Access which fosters wider collaboration and increased citations

- maximum visibility for your research: over $100 \mathrm{M}$ website views per year

At $\mathrm{BMC}$, research is always in progress.

Learn more biomedcentral.com/submissions 